\title{
Multiple high-pressure phase transitions in $\mathrm{BiFeO}_{3}$
}

\author{
Mael Guennou, ${ }^{1, *}$ Pierre Bouvier, ${ }^{1,2, \dagger}$ Grace S. Chen, ${ }^{1}$ Brahim Dkhil,${ }^{3}$ Raphaël Haumont ${ }^{4}$ \\ Gaston Garbarino, ${ }^{2}$ and Jens Kreisel ${ }^{1}$ \\ ${ }^{1}$ Laboratoire des Matériaux et du Génie Physique, CNRS, Grenoble Institute of Technology, MINATEC, 3 parvis Louis Néel, \\ F-38016 Grenoble, France \\ ${ }^{2}$ European Synchrotron Radiation Facility (ESRF), Boîte Postale 220, 6 Rue Jules Horowitz, F-38043 Grenoble Cedex, France \\ ${ }^{3}$ Laboratoire Structures, Propriétés et Modélisation des Solides, Ecole Centrale Paris, F-92290 Châtenay-Malabry, France \\ ${ }^{4}$ Laboratoire de Physico-Chimie de l'État Solide, ICMMO, CNRS, Université Paris XI, F-91405 Orsay, France
}

(Received 4 August 2011; published 11 November 2011)

\begin{abstract}
We investigate the high-pressure phase transitions in $\mathrm{BiFeO}_{3}$ by single-crystal and powder x-ray diffraction, as well as single-crystal Raman spectroscopy. Six phase transitions are reported in the 0-60-GPa range. At low pressures, four transitions are evidenced at 4, 5, 7, and $11 \mathrm{GPa}$. In this range, the crystals display large unit cells and complex domain structures, which suggests a competition between complex tilt systems and possibly off-center cation displacements. The nonpolar Pnma phase remains stable over a large pressure range between 11 and $38 \mathrm{GPa}$, where the distortion (tilt angle) changes only little with pressure. The two high-pressure phase transitions at 38 and $48 \mathrm{GPa}$ are marked by the occurrence of larger unit cells and an increase of the distortion away from the cubic parent perovskite cell. We find no evidence for a cubic phase at high pressure, nor indications that the structure tends to become cubic. The previously reported insulator-to-metal transition at $50 \mathrm{GPa}$ appears to be symmetry breaking.
\end{abstract}

DOI: 10.1103/PhysRevB.84.174107

PACS number(s): $81.40 . \mathrm{Vw}, 75.85 .+\mathrm{t}, 81.30 . \mathrm{Bx}$

\section{INTRODUCTION}

Magnetoelectric multiferroics currently attract a great deal of interest, both because of intriguing coupling mechanisms between magnetism, ferroelectricity, and ferroelasticity and because of the potential for new types of magnetoelectric devices. ${ }^{1-5}$ Among multiferroics, bismuth ferrite, $\mathrm{BiFeO}_{3}$ (BFO), is commonly considered as a model system ${ }^{6}$ and is perhaps the only material that is both magnetic and ferroelectric with a strong electric polarization at ambient conditions. Even though BFO attracts important attention, the crystal structures of BFO as a function of temperature and pressure are still debated in the literature. ${ }^{6}$

In addition to being a model multiferroic, $\mathrm{BFO}$ is also one of the very few perovskites that present both octahedral tilts $^{7-9}$ and strong ferroelectric cation displacements, thus two structural instabilities, at room temperature. Ab initio calculations suggest that in a number of perovskites both instabilities are present and compete. ${ }^{10,11}$ It is, however, important to remember that one instability usually prevails, so the vast majority of perovskites present either tilts $\left(\mathrm{SrTiO}_{3}, \mathrm{LaAlO}_{3}\right.$, etc.) or ferroelectric cation displacements $\left(\mathrm{BaTiO}_{3}, \mathrm{PbTiO}_{3}\right.$, etc.) at a given temperature or pressure, ${ }^{8}$ as schematized in Fig. 1. For some perovskites a crossover from one distortion to the other can occur as a function of an external parameter; $\mathrm{PbTiO}_{3}$ under high pressure is such an example. ${ }^{12} \mathrm{BiFeO}_{3}$ is thus a rather exceptional case and turns out to be a model system for studying the competition between tilts and polar instabilities in perovskites.

General rules for predicting phase transitions in perovskites have been of long-standing interest. As a fruit of past studies it is now generally accepted that increasing temperature reduces both tilt instabilities (the tilt angle decreases with increasing temperature) and polar instabilities (decrease of the polar cation displacement). ${ }^{8,13,14}$ As a consequence, increasing temperature drives perovskites toward the parent cubic perovskite, although the ideal cubic structure might be in some cases beyond the decomposition or melting point. The effect of high pressure on perovskites is more complex. Concerning octahedral tilts, it was originally suggested by Samara et al. ${ }^{15}$ that the phase transition temperatures $T_{c}$ of zone-boundary transitions in perovskites should always increase with pressure: $d T_{c} / d P>0$; i.e., the antiferrodistortive tilt angle increases with increasing pressure. However, experiments on $\mathrm{LaAlO}_{3}$ (Ref. 16) and later on other perovskites ${ }^{17-20}$ revealed that some perovskites decrease their tilt angle and undergo phase transitions to higher-symmetry phases on increasing pressure. As a consequence, Samara's rule has been extended by Angel et al. to a new general rule ${ }^{17}$ which predicts the behavior of octahedral tilts by taking into account the compressibilities of the different polyhedra, with so far no known exception.

As to polar instabilities, the pioneering work by Samara et al. ${ }^{15}$ describes that pressure reduces ferroelectricity in $A B \mathrm{O}_{3}$ perovskites and even annihilates it for a critical pressure $P_{c}$ at which the crystal structure becomes cubic. Early papers on $\mathrm{BaTiO}_{3},{ }^{21,22} \mathrm{KNbO}_{3},{ }^{23,24}$ and $\mathrm{PbTiO}_{3}$ (Refs. 25 and 26) amply confirmed this view and led to the widely accepted conclusion that polar perovskites adopt for $P>P_{c}$ a cubic $P m \overline{3} m$ crystal structure. It was thus unexpected to find out in recent years that relaxor ferroelectrics do not stay or become cubic at high pressure (e.g., Refs. 27-30) and that ferroelectric PZT displays a wealth of phase transitions under high pressure without becoming cubic either (e.g., Refs. 31-33). However, the most striking observation was that the model ferroelectric and simple perovskite $\mathrm{PbTiO}_{3}$ first reduces its ferroelectricity and becomes cubic but then, unlike commonly thought, becomes again noncubic with tetragonal phases ${ }^{12,34,35}$ through mechanisms involving oxygen octahedral tilting ${ }^{12}$ and a theoretically predicted reentrance of ferroelectricity. ${ }^{34-36}$

The aim of our study is to go beyond the above-discussed perovskites with one instability only by investigating the consequences of the competition of octahedral tilts and 


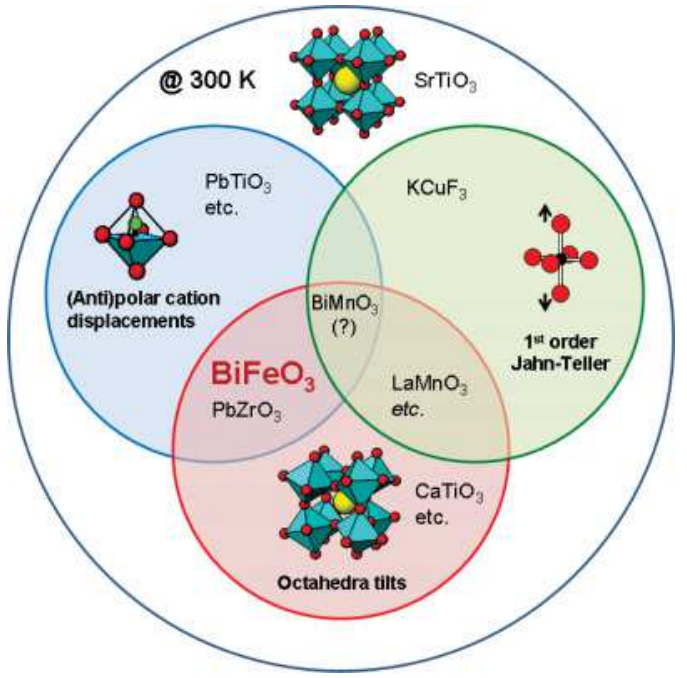

FIG. 1. (Color online) Schematic illustration of distortions and their combination in $A B X_{3}$ perovskites. Each of the three inner circles represents one of the three main collective structural distortions in perovskites: polar or antipolar cation displacements (top left blue shaded circle), octahedra tilts (bottom red shaded circle), and firstorder Jahn-Teller (top right green shaded circle) distortions, each illustrated by a schematic drawing and a material that adopts the distortion at ambient conditions. Materials outside the circles adopt the ideal cubic perovskite structure exemplified by $\mathrm{SrTiO}_{3} \cdot \mathrm{BiFeO}_{3}$ is among the very few materials that adopt at $300 \mathrm{~K}$ both strong ferroelectric cation displacements and strong octahedral tilts.

cation displacements on the crystal structure of $\mathrm{BiFeO}_{3}$, which is also magnetic. We focus on the effect of pressure, a parameter which allows driving to a very large extent structural instabilities, and thus their competition, thanks to large changes in cell volume and bond lengths unachievable otherwise. Despite literature reports on the effect of high pressure on BFO, the actual symmetries in the low-pressure regime remain controversial and the very high pressure regime has not been properly investigated (see Refs. 37-45 and the following section). Here, we investigate BFO by using Raman spectroscopy and synchrotron $\mathrm{x}$-ray diffraction on both powder and single crystals up to the very high pressure of $55 \mathrm{GPa}$, a regime only rarely explored in functional oxides save for some notable exceptions. . $^{2,39,46,47}$

We show that BFO undergoes a surprising richness of six phase transitions up to $55 \mathrm{GPa}$. In addition to this, we report for BFO unusually large and puzzling unit cells under high pressure, which are unexpected for simple $\mathrm{ABO}_{3}$ perovskites. The results are presented in light of the current literature on $\mathrm{BFO}$ and also in relation to other Bi-based perovskites.

\section{PRELIMINARY REMARKS}

The structure of $\mathrm{BFO}$ at high pressure has been investigated in the past in several studies, as schematized in Fig. 2. Powder $\mathrm{x}$-ray diffraction under high pressure has been performed over the past few years by several groups with various outcomes. Gavriliuk et al. ${ }^{42,48}$ and Zhu et al.$^{45}$ have reported no structural phase transition in this pressure range. Haumont et al. ${ }^{38}$ have identified two phase transitions at 3.5 and $10 \mathrm{GPa}$ with a proposed phase sequence $R 3 c \longrightarrow C 2 / m \longrightarrow P n m a$.



FIG. 2. (Color online) Schematic summary of the high-pressure investigations of BFO.

Belik et al. ${ }^{43}$ later confirmed the phase transition at 4 $\mathrm{GPa}$ and identified an additional transition at $7 \mathrm{GPa}$. They have identified the two intermediate phases as orthorhombic instead of the monoclinic $C 2 / \mathrm{m}$, with a transition sequence $R 3 c \longrightarrow$ Ortho I $\longrightarrow$ Ortho II $\longrightarrow$ Pnma, and also pointed out reversibility issues. In a previous work, we confirmed these transition pressures and emphasized the importance of good hydrostatic conditions. ${ }^{39}$ Very recently, Kozlenko et al. ${ }^{49}$ reported a powder neutron diffraction study and identified a high-pressure orthorhombic phase with space group Pbam between 3 and $8.6 \mathrm{GPa}$, distinct from the phases previously seen. In parallel, Raman spectroscopy has also been used to investigate phase transitions. ${ }^{37,44}$ Both studies revealed two phase transitions in the low-pressure range, but at somewhat different pressures, about 3 and $9 \mathrm{GPa}$. Theoretical studies have also been devoted to the high-pressure behavior of $\mathrm{BFO}^{50-53}$ and are commented on in the discussion.

Generally speaking, the determination of the structures of BFO from single-crystal diffraction in diamond-anvil cells (DACs) is complicated by (i) the limited access to reciprocal space, because of the geometry of the DACs, (ii) the complex domain structure that emerges as the crystal goes through the different phase transitions, and (iii) the very weak intensity of oxygen-related superstructure reflections as compared to the heavier Bi and Fe. Powder diffraction, on the other hand, is not limited by the DAC geometry, but overlapping bands, band broadening, and a stronger background hamper the observation of weak reflections and small splittings. Both techniques can be used in a complementary way, although single-crystal data give in general much better results, especially at high pressure. In this paper, we report four different diffraction experiments carried out on powder and single-crystal samples. The experiments are summarized in Table I with the pressure ranges investigated. In the following, the diffraction results are mostly taken from experiments 1 and 2 carried out with single crystals. Experiments 3 and 4 were carried out to check the reproducibility of the transitions; they are not presented in detail.

\section{EXPERIMENTAL DETAILS}

The BFO powder was prepared by conventional solid-state reaction using high-purity (better than 99.9\%) bismuth oxide $\left(\mathrm{Bi}_{2} \mathrm{O}_{3}\right)$ and iron oxide $\left(\mathrm{Fe}_{2} \mathrm{O}_{3}\right)$ as starting compounds. After 
TABLE I. List of the experiments reported in this paper with the pressure range investigated and the pressure-transmitting media (PTM) used.

\begin{tabular}{lcccc}
\hline \hline Expt. & Sample & PTM & Beam line & $P$ range (GPa) \\
\hline XRD 1 & Single crystal & Helium & ID09A & $0-12$ \\
XRD 2 & Single crystal & Neon & ID27 & $12-55$ \\
XRD 3 & Single crystal & Helium & ID27 & $30-55$ \\
XRD 4 & Powder & Helium & ID27 & $0-52$ \\
Raman & Single crystal & Alcohol & & $0-12$ \\
\hline \hline
\end{tabular}

mixing in stoichiometric proportions, powders were calcined at $T_{f}=820^{\circ} \mathrm{C}$ for $3 \mathrm{~h}$. BFO single crystals were grown by the flux method. Details about the synthesis can be found in Ref. 54. The single crystals used for the experiments were polished to a thickness of about $10 \mu \mathrm{m}$ with a lateral extension of 10 to $30 \mu \mathrm{m}$. Both optical inspection under polarized light and $\mathrm{x}$-ray diffraction (XRD) indicate that the crystals used were in a single domain state before the experiments.

All experiments were performed in DACs. The diamonds have the Boehler-Almax design with cullets of $600 \mu \mathrm{m}$ (experiment $1+$ Raman) or $250 \mu \mathrm{m}$ (experiments 2, 3, and 4). The pressure chamber was sealed by a stainless steel gasket. The pressure-transmitting media (PTM) used are given in Table I.

X-ray diffraction experiments were performed on the ID27 and ID09A beam lines at the ESRF. At ID27, the beam was monochromatic with a wavelength of $0.3738 \AA$ selected by an iodine K-edge filter and focused to a beam size of about $3 \mu \mathrm{m}$. At ID09A, the beam size is about $20 \mu \mathrm{m}$ and the wavelength $(0.4144 \AA)$ was determined from the calibration using a standard silicon powder. The signal was collected in the rotating crystal geometry on a marCCD 345 (ID27) or mar 555 image plate (ID09A) with $-30^{\circ} \leqslant \omega \leqslant 30^{\circ}$ in $0.5^{\circ}$ or $2^{\circ}$ steps. A precise calibration of the detector parameters was performed with a reference silicon powder. The pressure was measured by the standard ruby fluorescence method. ${ }^{55}$ The diffraction patterns from single-crystal measurements were indexed with a home-developed program based on the FIT2D software. ${ }^{56}$ The refinement of the lattice constants from the peak positions was performed with the program UNITCELL. ${ }^{57}$ The CRYSALIS software (Oxford Diffraction-Agilent) was used for reconstruction of planes in reciprocal space. For the powder experiment, a gold powder was used as a pressure standard. ${ }^{58}$ The pressure calculated from the gold standard was corrected for nonhydrostatic effects by following the procedure described by Singh. ${ }^{59}$

The Raman spectra were recorded on a Jobin-Yvon Labram spectrometer with a low-frequency cutoff at $100 \mathrm{~cm}^{-1}$. The exciting laser line was $633 \mathrm{~nm}$. The laser power was kept at $10 \mathrm{~mW}$ on the DAC to avoid heating of the sample. The spectra were unpolarized.

\section{RESULTS}

The analysis of the diffraction patterns reveals six phase transitions at $4,5,7,11,38$, and $48 \mathrm{GPa}$ with the phase sequence $R 3 c \longrightarrow$ Phase II $\longrightarrow$ Phase III $\longrightarrow$ Phase IV $\longrightarrow$ Pnma $\longrightarrow$ Phase VI $\longrightarrow$ Phase VII. The $R 3 c$ and Pnma phases were reported previously and are only confirmed by our experiments. In contrast, the structure of phases II, III, IV, VI, and VII have not been determined. In the following, we divide the results into two distinct pressure ranges: the low-pressure region from ambient pressure up to $15 \mathrm{GPa}$ and the high-pressure region for the Pnma phase and above. Finally we give the evolution of the lattice constants, volume, and strains over the full pressure range.

\section{A. Low-pressure region: 0-15 GPa}

An overview of the experimental evidence for the four phase transitions in the low-pressure range is given in Fig. 3, where we present the integrated single-crystal diffraction pattern (left) and the Raman spectra (right).

The transition from the low-pressure $R 3 c$ phase to phase II is marked by the emergence of new superstructure reflections (blue in Fig. 3). In addition, splittings of diffraction peaks show the emergence of a complex domain structure. A simple observation under a microscope also shows that the single domain has split into a multitude of very small domains in phase II.

At $5 \mathrm{GPa}$, we observe the disappearance of many diffraction peaks, revealing a simplification of the domain structure, and a marked change of the Raman signature, marking the transition from phase II to phase III (orange in Fig. 3). The transition from phase III to phase IV (black in Fig. 3) at $7 \mathrm{GPa}$ is not characterized by any pronounced change in the diffraction pattern in terms of emergence or disappearance of diffraction peaks. A close inspection of the Raman spectra does reveal small changes at $7 \mathrm{GPa}$, notably at low wave numbers with the emergence of a mode at $100 \mathrm{~cm}^{-1}$. The most clear signature of this transition are sudden shifts of diffraction peaks at low $2 \theta$ toward lower angles, which can only be associated with sudden changes in the lattice constants. This change coincides well with the transition seen at the same pressure in the powder diffraction experiment by Belik et al. ${ }^{43}$ At $11 \mathrm{GPa}$, we observe

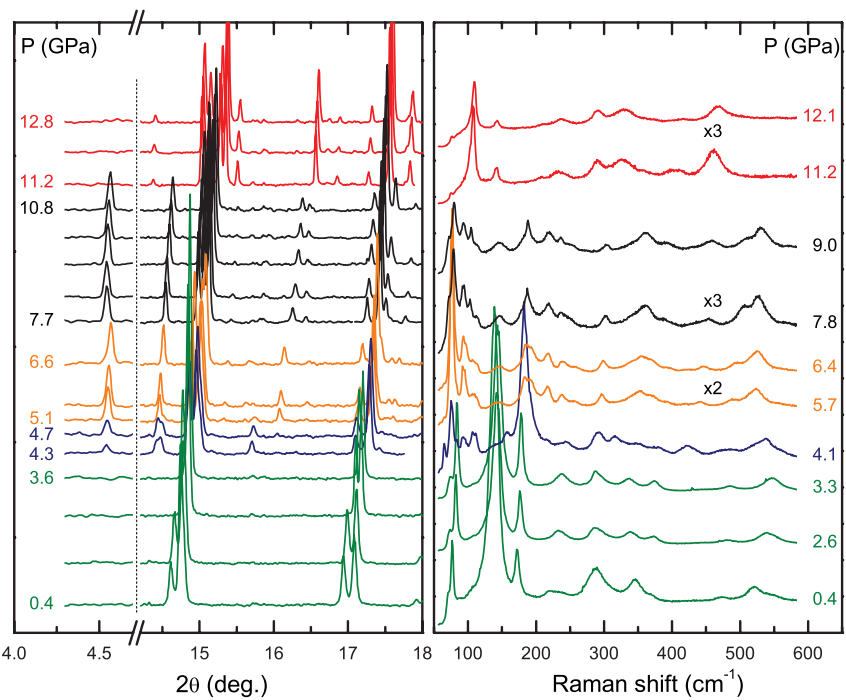

FIG. 3. (Color online) (left) Selected parts of the integrated diffraction patterns of the single crystal for the low-pressure experiment $(\lambda=0.4144 \AA$ ) with phases $R 3 c$ (green), II (blue), III (orange), IV (black), and Pnma (red). (right) Raman spectra up to $12.1 \mathrm{GPa}$. 
$5.5 \mathrm{GPa}$

$a=5.484(1) A \quad b=16.674(1) A \quad c=7.737(1) A$
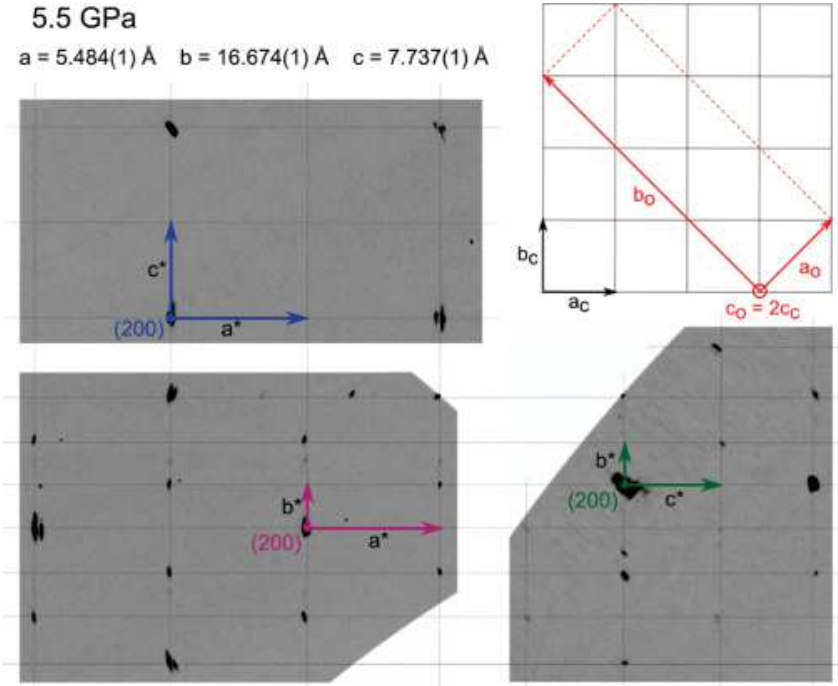

FIG. 4. (Color online) Maps of $(h k 0),(2 k l)$, and $(h 0 l)$ reciprocal planes obtained at 5.5 GPa. Due to the orientation of the sample in the DAC, we have almost no access to the $(0 k l)$ plane and the sampling for $(2 k l)$ is less precise. The relations of the orthorhombic unit cell axes to the simple cubic axes are recalled.

the transition to the Pnma phase by changes in both the diffraction patterns and the Raman signature (red in Fig. 3).

The diffraction patterns for all three phases can be indexed with an orthorhombic unit cell, taking into account the complex domain structure. This cell has lattice contants $a_{\mathrm{o}}=5.484(1) \AA, b_{\mathrm{o}}=16.674(1) \AA$, and $c_{\mathrm{o}}=7.737(1) \AA$ at $5.5 \mathrm{GPa}$ and a comparatively large volume $(Z=12)$. For this cell, pseudocubic lattice constants can be calculated as $a_{\mathrm{pc}}=a_{\mathrm{o}} / \sqrt{2}, b_{\mathrm{pc}}=b_{\mathrm{o}} /(3 \sqrt{2})$ and $c_{\mathrm{pc}}=c_{\mathrm{o}} / 2$. In Fig. 4, we reproduce maps of reciprocal space at $5.5 \mathrm{GPa}$ and give the relations between the lattice vectors of the orthorhombic cell and those of the parent cubic perovskite.

Metrically, this cell is close to the orthorhombic cell proposed by Belik et al. ${ }^{43}$ except that we find a $c$ axis doubled in all phases, as demonstrated at $5.5 \mathrm{GPa}$ by the presence of the $(2 k 1)$ reflections in Fig. 4 . The pattern can also be indexed with the $C 2 / \mathrm{m}$ phase proposed by Haumont $e$ t al., ${ }^{38}$ but the diffraction does not give evidence for a monoclinic distortion. On the other hand, the orthorhombic cell identified by Kozlenko et al. in their neutron diffraction study, ${ }^{49}$ with lattice constants $\sqrt{2} a_{\mathrm{pc}} \times 2 \sqrt{2} a_{\mathrm{pc}} \times 2 a_{\mathrm{pc}}$, cannot explain the observed patterns.

The inspection of the reflections in Fig. 4 shows the typical conditions for $I$ centering. In addition, the inspection of the $(h 0 l)$ plane shows that only reflections with $h, l=2 n$ are present. This leaves the possibility for two extinction symbols $I-(a c)$ - and $I-c b$, which we cannot distinguish here because of an insufficient coverage of the $h k 0$ plane due to the DAC geometry. This leads to six different possibilities among four space groups: Ima2 (46), I2cm (46), I2cb (45), Imam (74), Imcm (74), and Imcb (72).

Within this choice of space group assignments, we can tentatively infer information from the evolution of the domain structure at the transition II $\longrightarrow$ III. For a ferroic transition between two orthorhombic space groups, the formation of twins can only occur for a transition from point group $\mathrm{mmm}$ for the parent phase to $m m 2$ or $222 .^{60}$ The simplification of the domain structure therefore suggests that phase II has a space group Ima2, I2cm, or $I 2 c b$ while phase III has Imam, Imcm, or Imcb. For the transition III $\longrightarrow$ IV, on the other hand, the diffraction pattern does not show any sign of a symmetry change or any change in the domain structure. This leaves the possibility of a transition between any of the three centrosymmetric space groups mentioned above, which includes isostructural transitions.

\section{B. High-pressure region: 11-60 GPa}

In the high-pressure region, we report two phase transitions at 38 and $48 \mathrm{GPa}$. Each phase is separately described. Finally, we discuss the issue of nonhydrostatic stress conditions.

\section{Phase V: 11-38 GPa (Pnma)}

Between 11 and $38 \mathrm{GPa}$, we find the now well-established $\mathrm{GdFeO}_{3}$-type Pnma phase. A representative diffraction pattern is shown in Fig. 5. In this phase, the crystal shows peak splitting associated with a four-variant domain structure, as is commonly observed for Pnma crystals. As the pressure

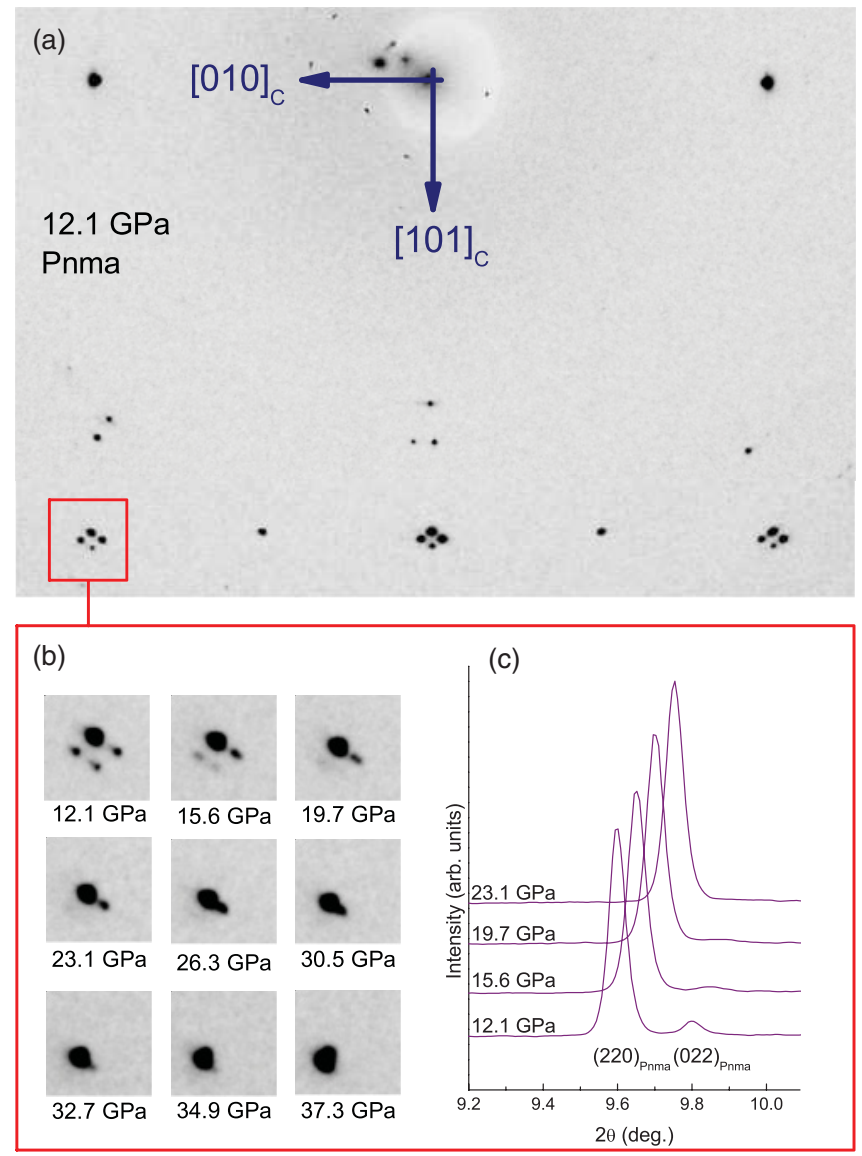

FIG. 5. (Color online) (a) Diffraction pattern of a $\mathrm{BiFeO}_{3}$ single crystal in the Pnma phase integrated over the full angular range. The peaks show a splitting associated to the domain structure. (b) Evolution of the $(222)_{C}$ reflection under pressure and (c) selected corresponding $2 \theta$ scans. 
increases, however, the intensity ratio changes and reflects the formation of a quasi-single-domain state [Figs. 5(b) and 5(c)].

\section{Phase VI: 38-48 GPa}

At $38 \mathrm{GPa}$, additional sets of superstructure appear as can be seen in Fig. 6 on the reciprocal space map of the $(h k 0)$ plane. Metrically the new cell can be derived from the Pnma cell by a doubling of the $a$ axis. At $43 \mathrm{GPa}$, we find $a_{\mathrm{o}}=10.546(4) \AA$, $b_{\mathrm{o}}=7.393(3) \AA$, and $c_{\mathrm{o}}=5.113(16) \AA$. For this phase, we have $Z=8$ and we calculate pseudocubic lattice constants as $a_{\mathrm{pc}}=a_{\mathrm{o}} /(2 \sqrt{2}), b_{\mathrm{pc}}=b_{\mathrm{o}} / 2$, and $c_{\mathrm{pc}}=c_{\mathrm{o}} / \sqrt{2}$. Note that diffraction spots of the (100) reflections are clearly visible, but (200) are not seen, which can only be explained by their weak intensity. Also, in this pattern, a small portion of a second crystal is visible, but it is disoriented with respect to the main crystal, which may indicate that it comes from a broken piece of the crystal rather than a phase-transition-induced twin. The intensity of this additional peak is, however, much weaker than the Bragg peaks of the main crystal and makes any confusion unlikely.

The analysis of the reflection conditions leads to two possible extinction symbols $(\mathrm{Pb}--$ and $\mathrm{Pn}--)$. These two possibilities differ by conditions $0 k l: k$ and $0 k l: k+l$, respectively. Unfortunately, our collected single-crystal pattern does

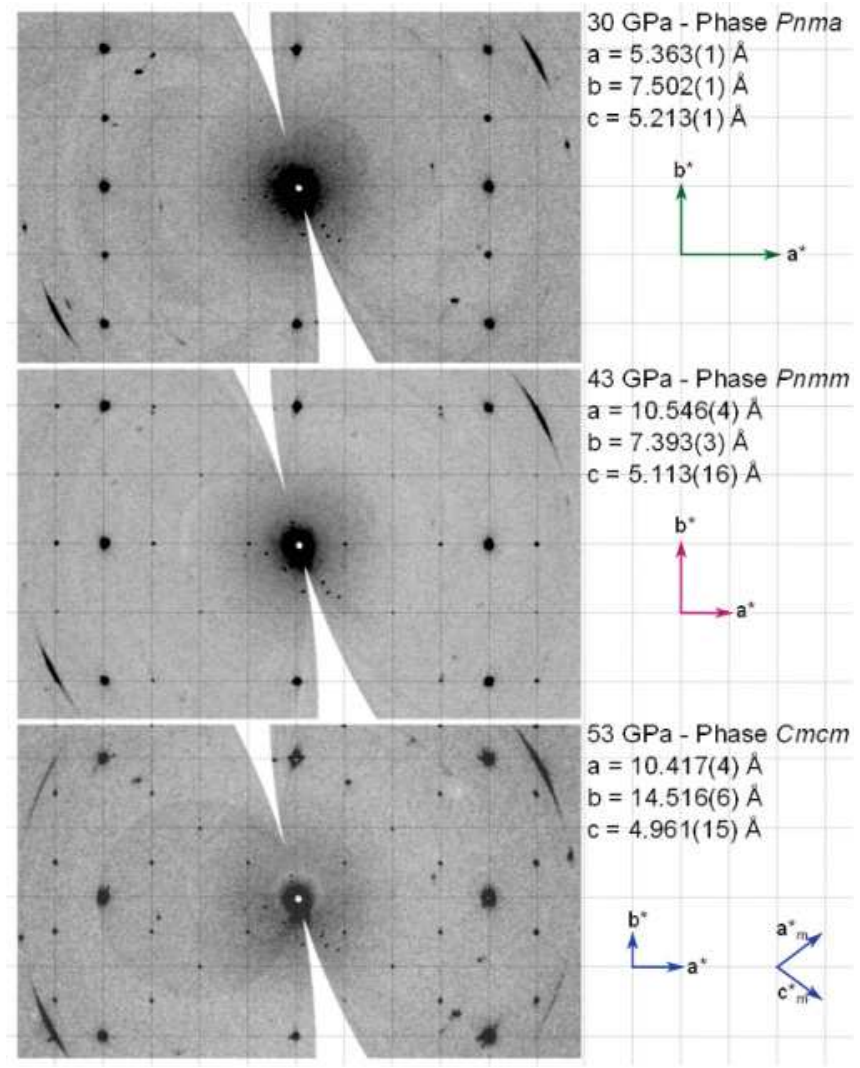

FIG. 6. (Color online) Reciprocal space maps of the $(h k 0)$ plane at $30 \mathrm{GPa}$ (phase Pnma), $43 \mathrm{GPa}$ (phase Pnmm), and $53 \mathrm{GPa}$ (phase $\mathrm{Cmcm}$ ), taken from experiment 2 (neon as PTM). The images have been rescaled for convenience so that the overall volume reduction is not apparent. The lattice constants and reciprocal lattice vectors for the different cells are recalled, including the monoclinic axes for the very last cell. not allow to distinguish the two possibilities because of the restricted coverage of the $0 \mathrm{kl}$ plane due to the experimental geometry and the orientation of the crystal in the cell. We found that the weak (100) reflections, although unambiguous in the single-crystal pattern, cannot be seen in the powder diffraction pattern. The ratio of the intensities of these weak superstructures to the main Bragg peaks is about 1/10 000 . Powder diffraction alone would fail to identify the correct unit cell and did not allow to discriminate between the two possibilities $P b--$ and $P n--$. We are left with five possible space groups: the three polar groups $P b 2_{1} m$ (26), $P b m 2$ (28), and $P n m 2_{1}$ (31) and two nonpolar groups Pbmm (51) and Pnmm (59). Based on the generally accepted fact that ferroelectricity vanishes under pressure and that there is no known ferroelectric perovskite beyond $15 \mathrm{GPa}$, we consider the last two possibilities as the most probable. In addition, we note that the Pnmm opens the possibility for a group-subgroup relation with the Pnma phase.

\section{Phase VII: P > $48 \mathrm{GPa}$}

The transition from phase VI to phase VII is evidenced by a clear change in the diffraction pattern. A reciprocal space map is presented in Fig. 6. This pattern can be indexed in a $C$-centered orthorhombic cell with the same axis directions as in phase VI. This new orthorhombic cell is derived from the Pnmm cell by a doubling of the $b$ axis, leading to lattice constants $a=10.417(4) \AA, b=14.516(6) \AA$, and $c=4.961(15) \AA$ at $53 \mathrm{GPa}$, with 16 formula units per unit cell. With this indexing, the analysis of the reflection conditions leads to the extinction symbol $C-c-$, which allows for three different space groups: $C m c 2_{1}$ (36), $C 2 \mathrm{~cm}$ (40), and $C \mathrm{mcm}$ (63). The first two space groups are polar. With the same argument as before, we regard $\mathrm{Cmcm}$ as the most probable space group for this phase.

Alternatively, the pattern can be indexed in a monoclinic cell. This cell has a volume half of the orthorhombic cell $(Z=$ 8) and reciprocal lattice vectors related to the orthorhombic vectors by $\mathbf{a}_{M}^{*}=\mathbf{a}_{O}^{*}+\mathbf{b}_{O}^{*}, \mathbf{c}_{M}^{*}=\mathbf{a}_{O}^{*}-\mathbf{b}_{O}^{*}$, and $\mathbf{b}_{M}^{*}=\mathbf{c}_{O}^{*}$, as depicted in Fig. 6. The orthorhombic and monoclinic cells are equivalent in the limit $\mathbf{a}_{M}^{*}=\mathbf{c}_{M}^{*}$. The analysis of the extinctions leave the possibilities for five monoclinic space groups for which no reflection condition applies: $P 2$ (3), $P 2_{1}$ (4), $P m$ (6), $P 2 / m$ (10), and $P 2_{1} / m$ (11). Discarding as before the polar space groups, the two space groups $P 2 / m$ and $P 2_{1} / m$ appear as more likely.

\section{Importance of nonhydrostatic stress}

As long as the PTM remains liquid $(P<12 \mathrm{GPa}$ for $\mathrm{He}$, $4 \mathrm{GPa}$ for $\mathrm{Ne}$, and $10 \mathrm{GPa}$ for the classical 4:1 methanolethanol mixture), the stress field can be safely regarded as hydrostatic. However, above this limit, a so-called deviatoric component may add to the hydrostatic stress field. The deviatoric stress has a rotational symmetry around the loading axis of the cell, with a stronger compressive stress along the axis, as checked by Zhao et al. ${ }^{61}$ in an experiment with silicon oil as the PTM. Nonhydrostatic stress should then be regarded as unavoidable at high enough pressure, even though they are very often (legitimately) neglected when noble gases, particularly helium, are used as PTM. It is nonetheless 
of concern, for a meaningful comparison between different experimental works and with ab initio calculations, to consider how such nonhydrostatic stress affects the outcome of an experiment. This can be done, for example, by comparing experiments performed in different PTM or, for single-crystal investigations, by comparing experiments with crystals of different orientations.

In a previous diffraction work on $\mathrm{BiFeO}_{3}$ single crystals in the $0-10-\mathrm{GPa}$ range, we have shown that strongly nonhydrostatic stress has a remarkable effect on the phase transitions by stabilizing a structure that is not observed in hydrostatic conditions. ${ }^{39}$ Here, we have shown an evolution of the domain structure in the Pnma phase (Fig. 5), which can be explained by the onset of nonhydrostatic stress favoring one particular domain orientation. However, we also make the following observations. First, the same phases were observed in two experiments performed with different pressure-transmitting media (helium versus neon). Moreover, the powder diffraction experiment shows the same transitions, although with slightly different pressures. Therefore, we believe that nonhydrostaticity of the stress field plays a minor role here and is only likely to shift transition pressure and affect slightly the evolution of the lattice constants, but without affecting the structures.

In addition, complications may arise from the ferroelastic character of BFO. As the crystal undergoes different phase transitions, ferroelastic domain formation and reorientation may act as stress relief and modify the stress state of the sample. This can make hydrostatic conditions difficult to be achieved, even at low pressures. Ferroelasticity in BFO is unfortunately not extensively studied in the literature but deserves further attention in light of the above comments.

\section{Volume, lattice constants, and strain}

In Fig. 7, we present the evolution of the lattice constants, volume, and strain over the full pressure range. The strain is calculated as follows. As a reference state for all the phases, we choose a cubic cell with lattice constant $a_{0}=(V / Z)^{1 / 3}$. The general expressions for the strain components $e_{i j}$ can then be found in Ref. 62. In the orthorhombic system, the expressions reduce to $e_{11}=a_{\mathrm{pc}} / a_{0}-1, e_{22}=b_{\mathrm{pc}} / a_{0}-1$, and $e_{33}=c_{\mathrm{pc}} / a_{0}-1$, where $a_{\mathrm{pc}}, b_{\mathrm{pc}}$, and $c_{\mathrm{pc}}$ are the pseudocubic lattice constants. We then calculate the "total" strain using Aizu's expression $e_{\text {tot }}=\sqrt{\sum e_{i j}^{2}}$. This choice is somewhat arbitrary, but it enables us to quantify and compare the strain in the different phases.

In the evolution of the lattice constants, the most remarkable feature is the transition III $\longrightarrow$ IV that is marked by a "switching" of the $a_{\mathrm{pc}}$ and $c_{\mathrm{pc}}$ parameters for a volume (and a strain) that shows no significant jump, as already reported by Belik et al. in their powder diffraction study. ${ }^{43}$ We have no precise description of the microscopic mechanism underlying this phase transition, but we note that a behavior of lattice constants similar to the present case was observed in $\mathrm{BiMnO}_{3}$ (Ref. 63) under pressure, where it was associated to a change in the magnetic order.

The evolution of the strain reveals the different distortion of the successive phases. With increasing pressure, the structure tends first toward a metrically cubic structure, whereby the

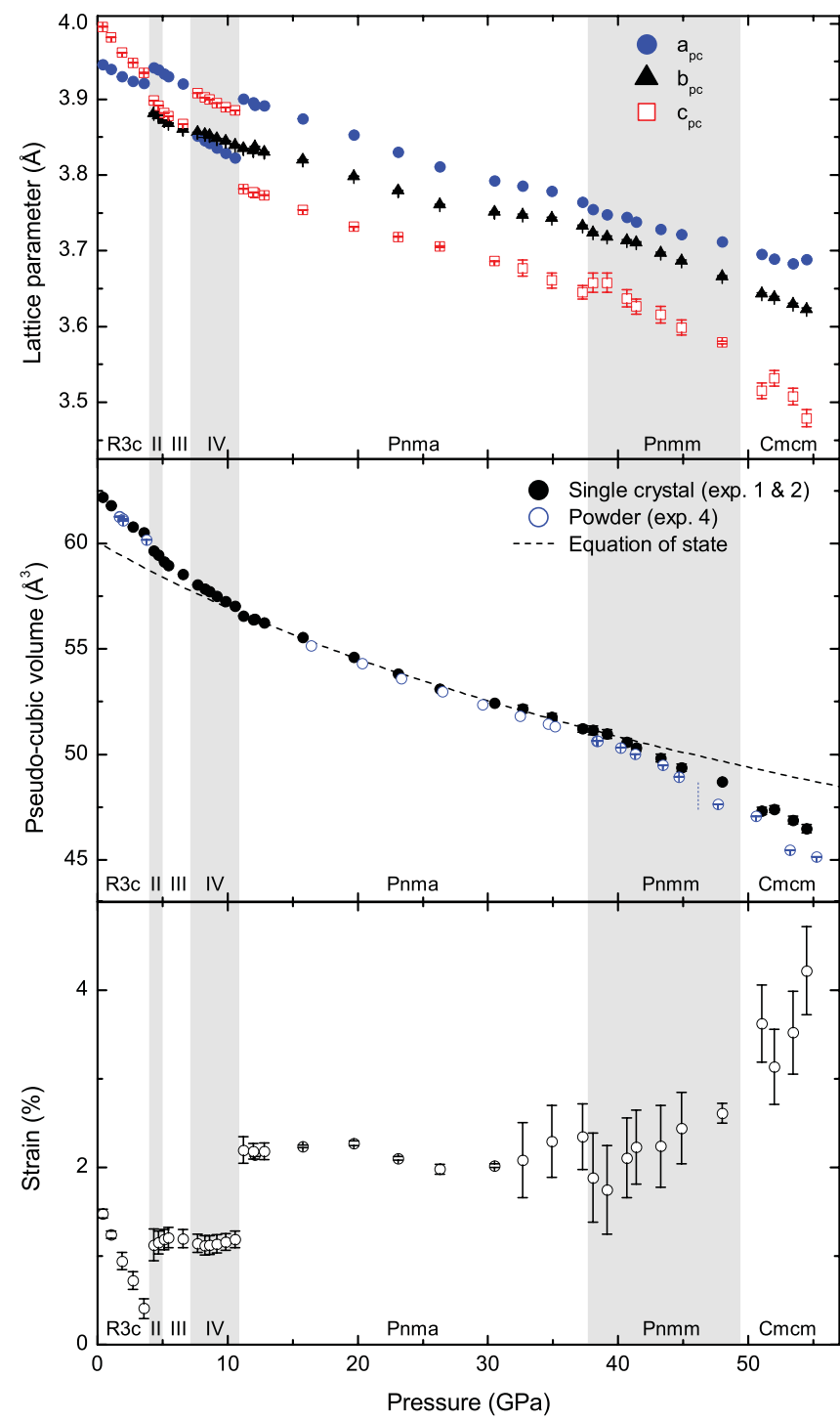

FIG. 7. (Color online) Evolutions of the (top) pseudocubic lattice constants, (middle) volume, and (bottom) total strain $e_{\text {tot }}$ in the full pressure range investigated. Data are from the single-crystal experiments 1 and 2 . In the volume plot, the data from the powder diffraction experiment have been added for comparison, and the volume calculated from the equation of state determined for the Pnma phase has been extrapolated over the full pressure range as a guide to the eye.

strain approaches zero. This evolution is in good agreement with the literature. ${ }^{38}$ The strain does not change appreciably through the transitions II $\longrightarrow$ III $\longrightarrow$ IV. Then, it shows a jump at the transition IV $\longrightarrow P n m a$, which appears more distorted than the original $R 3 c$ structure, and remains constant within experimental uncertainty through the Pnma stability range. At higher pressures, above $40 \mathrm{GPa}$, the tendency of an increase of the distortion away from a cubic metricity is very clear.

In the volume plot, we have also plotted the values determined from the powder XRD experiment. The volume measured from the powder appears to deviate slightly from the values measured on the single crystal, which might be due to the different pressure-transmitting media used 
(helium versus neon). A fit of the pseudocubic volume in the stability region of the Pnma phase with a second-order Birch-Murnaghan equation leads to $V_{12 \mathrm{GPa}}=56.43(8) \AA^{3}$ and $K_{12 \mathrm{GPa}}=218(5) \mathrm{GPa}$ for $K_{12 \mathrm{GPa}}^{\prime}$ being fixed to 4 . This value compares reasonably well with the previously published values of $238 \mathrm{GPa}^{38}$

Last, we discuss the order of the transitions. From the discontinuities in the volume and lattice constants, it is clear that the transitions $R 3 c \rightarrow \mathrm{II}, \mathrm{III} \rightarrow \mathrm{IV}$, and IV $\rightarrow$ Pnma at 4, 7, and $11 \mathrm{GPa}$, respectively, are of first order. Transitions $\mathrm{II} \rightarrow \mathrm{III}$ and Pnma $\rightarrow$ Pnmm at 5 and $38 \mathrm{GPa}$, on the other hand, present possible group-subgroup relationships and show no appreciable volume discontinuities, rather suggesting second-order transitions. This also seems to be the case for the last transition $P n m m \rightarrow C m c m$ at $48 \mathrm{GPa}$, although it is difficult to be conclusive due to the uncertainty of the volume measurements and the limited number of points close to the transition.

\section{DISCUSSION}

\section{A. Multiple phases of BFO}

Perovskites are known for their richness of structural and physical phase transitions with different possible instabilities which can coexist and compete. Both a fundamental and a technological interest derive from this characteristic. One consequence of the various instabilities is an often complex phase diagram of perovskites, for instance in the $P-T$ or $x-T$ spaces, which are the most explored diagrams. Besides perovskite-type manganites, ferroelectric perovskites are among the most investigated materials with the representative examples $\mathrm{PbZr}_{1-x} \mathrm{Ti}_{x} \mathrm{O}_{3}(\mathrm{PZT})$ for the $x-T$ space ${ }^{64-66}$ and $\mathrm{BaTiO}_{3}$ (BTO) or $\mathrm{KNbO}_{3}$ (KNO) for the $P-T$ space. ${ }^{24,67}$ Both examples display complex phase diagrams. Nevertheless, it is important to realize that when only one parameter $(P, T$, or $x$ ) is changed, no more than three phase transitions are observed, e.g., the rhombohedral-orthorhombic-tetragonal-tocubic phase sequence in BTO or KNO under pressure. ${ }^{24,67}$ Most other materials display fewer phase transitions under pressure: e.g., $\mathrm{CaTiO}_{3}$ (no transition ${ }^{47}$ ), $\mathrm{SrTiO}_{3}$ (one transition ${ }^{46}$ ), or $\mathrm{PbTiO}_{3}$ (two transitions ${ }^{12}$ ). In light of this, the six phase transitions of $\mathrm{BiFeO}_{3}$ observed here are remarkable. It is plausible to relate this observation to the fact that BFO presents at ambient conditions the rare coexistence of octahedral tilts, cation displacements, and magnetic order.

A recent study by Diéguez et al ${ }^{52}$ reinforces this intuitively expected intrinsic richness of structural phases in BFO by performing a systematic search for potentially stable phases by using first-principles methods. As a major outcome of their work, it was demonstrated that BFO can present an unusually large number of (meta)stable structures of which the balance or stability is determined not only by the traditional soft modes but also by secondary modes. Our observation of a pressure-induced rich phase sequence corroborates experimentally the predicted structural richness of BFO. However, our experimental results go beyond the predictions in the sense that we report new phases with unusually large cell dimensions, illustrating an even more complex energy landscape than suggested by Diéguez et al.'s calculations, ${ }^{52}$ which were restricted for computational reasons to smaller cells. The physical origin of such large unit cells is not yet understood, although its systematic occurrence in the low-pressure regime of $\mathrm{Bi}$-based perovskites such as $\mathrm{BiMnO}_{3}$, $\mathrm{BiScO}_{3}$, and $\mathrm{BFO}$ has been hypothetically related to the presence of the so-called lone pair in bismuth (see discussion in Ref. 38). We note, however, that we have shown here that BFO presents such large cells also at very high pressure, in sharp contrast to $\mathrm{BiMnO}_{3}$, where the unit cells at very high pressure are small. ${ }^{68}$ This suggests that the mechanism for very large unit cells has to be understood beyond the simple presence of $\mathrm{Bi}$ and thus deserves further attention, namely through adapted first-principles calculations. $\mathrm{NaNbO}_{3}$ (Ref. 69) and $\mathrm{AgNbO}_{3}$ (Ref. 70) show similarly large unit cells that have been interpreted in terms of complex tilt systems. Here, we understand by "complex tilt systems" collective tilts of the oxygen octahedra, whose description requires more than a doubling of the lattice constants of the cubic primitive perovskite cell and as such goes beyond the now classical approach by Glazer. ${ }^{7,71}$ These compounds also show a coexistence between octahedral tilts and (anti)ferroelectric cation ordering and might provide some understanding.

\section{B. Stability and compressibility of the Pnma phase}

A particular phase within this complex structural landscape is the orthorhombic Pnma phase. Generally speaking, Pnma is one of the very common structures among perovskites and is known to withstand very high pressures. In particular, the rare-earth orthoferrites $R \mathrm{FeO}_{3}$ maintain their Pnma structure at high pressure, even through their large volume collapse associated with a spin transition around $50 \mathrm{GPa}^{72}$ In BFO, it presents a remarkable stability from $11 \mathrm{GPa}$ up to $38 \mathrm{GPa}$. This contrasts with the effect of high temperature, where it persists only in a narrow temperature range. ${ }^{73}$ The stability is in agreement with calculations ${ }^{52}$ that have predicted that the Pnma phase constitutes BFO's most stable phase in addition to the room-temperature $R 3 c$ phase.

In the Pnma phase, the evolution of the tilt angles under pressure is of particular interest due to the importance of these angles for the electronic band structure. The two tilt angles Ti-O1-Ti $\left(\alpha_{1}\right)$ and Ti-O2-Ti $\left(\alpha_{2}\right)$ that characterize the Pnma structure ${ }^{8}$ are best determined from the atomic positions obtained by Rietveld refinements, but the quality of our powder data does not allow to determine atomic position with satisfying uncertainties. Tilt angles can also be calculated from the lattice constants under the assumption of undistorted polyhedra, ${ }^{8}$ but these formulas are known to give only rough estimations of the tilt angles. ${ }^{47}$ From these formulas and our measured lattice constants, we can estimate that the octahedral tilt angles decrease in the Pnma structure by less than $2^{\circ}$ between 10 and $38 \mathrm{GPa}$, with only little changes in the spontaneous strain (Fig. 7).

Following Angel et al. compressibility rules, ${ }^{17}$ the tilt angle is predicted to decrease with increasing pressure for most $A^{3+} B^{3+} \mathrm{O}_{3}$ perovskites, while it increases for $A^{2+} B^{4+} \mathrm{O}_{3}$ perovskites. Our experimental observation appears in qualitative agreement with this rule, in spite of the presence of the lone pair of bismuth. Such small changes have been reported ${ }^{19}$ to occur in orthoferrites in the presence of large and heavy $A$ 
cations, as is the case here for BFO. In future works, it would be interesting to investigate in more detail rare-earth orthoferrites in a similar manner to obtain a better understanding of the particular role of bismuth on the compressibility of the perovskite.

\section{Implications for the insulator-to-metal transition}

Another aspect of the very high-pressure regime is the reported occurrence of an insulator-to-metal (MI) phase transition accompanied by the disappearance of magnetic order around $50 \mathrm{GPa} .{ }^{40-42,48}$ There is an ongoing discussion in the literature on the origin of this MI transition in terms of a high-spin to low-spin crossover, a Mott-type transition, and changes in crystal symmetry being related or not. $6,48,53,74,75$ Our structural study does naturally not allow discussing in detail the proposed models of these physical phase transitions. However, our study provides evidence that the MI transition is symmetry breaking, and it rules out a transition to the earlier considered cubic $^{6}$ or rhombohedral ${ }^{48,53}$ phases. Rare-earth orthoferrites $R \mathrm{FeO}_{3}$ present a similar transition around $50 \mathrm{GPa}$ which has been assigned to a low-to-high spin phase transition of the $\mathrm{Fe}^{3+}$ cations accompanied by a steep anomaly in the cell volume, particularly marked in $\mathrm{LuFeO}_{3} .{ }^{72,76}$ Such a steep anomaly, characteristic for low-to-high spin phase transition is not observed in our measurements, thus rather questioning such a scenario.

\section{CONCLUDING REMARKS}

In this study we have presented a structural investigation of the model multiferroic perovskite $\mathrm{BiFeO}_{3}$ under high pressure up to $60 \mathrm{GPa}$. The complementary use of synchrotron $\mathrm{x}$-ray diffraction and Raman scattering reveals a remarkable richness of six phase transitions in the investigated pressure range. The occurrence of these six pressure-induced phase transitions together with the four transitions as a function of temperature reported in the literature ${ }^{6,52,77,78}$ suggests a very complex $P-T$ phase diagram that is characterized by the competition of the BFO-characteristic instabilities: octahedral tilts, cation displacements, magnetic order, and MI phase transitions.

In a first attempt, Catalan and $\mathrm{Scott}^{6}$ suggested in 2009 a preliminary and very useful phase diagram. Since then, our understanding of the different phase transitions has increased and we propose in Fig. 8 an updated schematic phase diagram, which is based on both literature results and the outcome of our present pressure work. In the following, we discuss the different features of this phase diagram with a particular emphasis on the essential points of our present work.

(i) In agreement with the calculations by Diéguez et al., ${ }^{52}$ the phase diagram is characterized by two particularly stable phases. First is the rhombohedral $R 3 c$ phase (orange in Fig. 8), which is at ambient pressure stable over $1000 \mathrm{~K}$. Despite this temperature stability, the $R 3 c$ structure is rapidly destabilized under hydrostatic pressure and disappears at a modest pressure of $4 \mathrm{GPa}$. Second is the orthorhombic Pnma structure (blue), which is stable at $300 \mathrm{~K}$ between 11 and $38 \mathrm{GPa}$. We have shown that the Pnma phase is characterized by only small changes in the spontaneous deformation (and octahedral tilts), so the compression mainly acts through bond shortening.

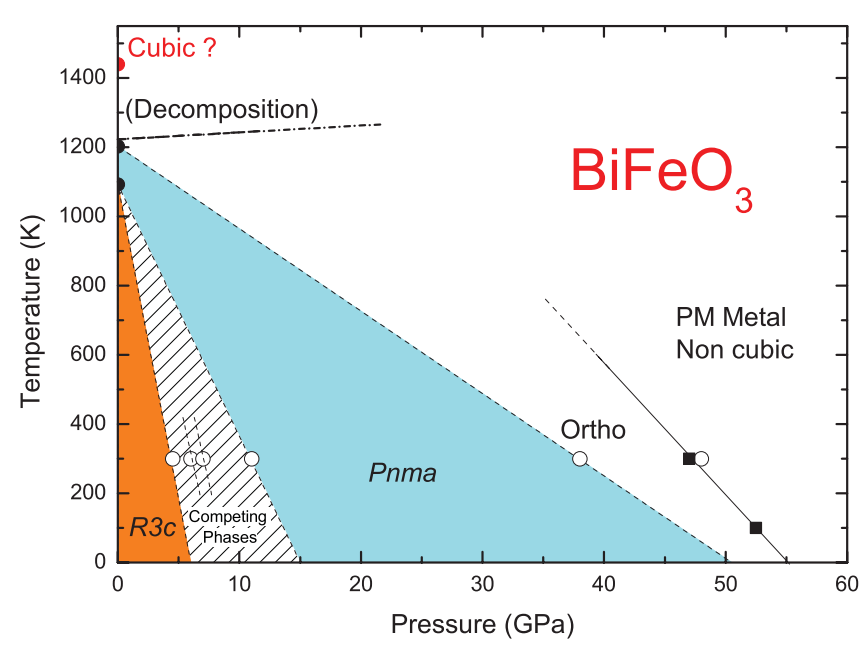

FIG. 8. (Color online) Revised schematic pressure-temperature phase diagram of BFO. The symbols are experimental points taken from this work (open symbols) and Refs. 73 and 73 (solid symbols). The transition temperature to a cubic phase at ambient pressure lies beyond the decomposition temperature and is a theoretical prediction. ${ }^{79}$

(ii) Between the stable $R 3 c$ and Pnma phases, we observe under high pressure three different phases of orthorhombic symmetry and thus a rather complex energy landscape within only a few GPa. All three intermediate phases are characterized by unusually large unit cells, implying complex tilt systems and possibly off-center cation displacements triggered by the lone pair of bismuth. In the phase diagram we consider this intermediate region (striped in Fig. 8) as only one single region that is characterized by a multitude of competing phases, which might well reveal even more richness when varying the parameter temperature. The suggestion of a distinct antipolar phase by Kozlenko et al. ${ }^{49}$ supports this view. We also remind that new phases can easily be induced by nonhydrostatic conditions in this region. ${ }^{39}$ The phase diagram is consistent with the idea of a complex deformation-temperature phase diagram, as can be also explored by temperature-dependent investigation of strained thin films (although the biaxial strain in thin films can of course not be directly compared to hydrostatic conditions). The very recent reports ${ }^{80-83}$ of a structural phase transition close to room temperature in highly compressively strained BFO thin films adds further support to this.

(iii) The very high pressure regime is characterized by two further phase transitions at 38 and $48 \mathrm{GPa}$, which are again characterized by large unit cells. Interestingly, we observe in this regime an increase of the distortion away from the cubic parent perovskite cell, which contrasts the earlier sketched ${ }^{6}$ high-pressure tendency toward a cubic structure. On the other hand, this is consistent with ab initio calculations by GonzálezVázquez and Íñiguez, according to which the simple cubic phase does not become the ground state even at high pressure. ${ }^{53}$ At high temperature, the most recent studies ${ }^{73}$ show that the cubic phase can only be expected at temperatures higher than the decomposition point and is therefore regarded as unstable.

(iv) The insulator-to-metal phase transition in $\mathrm{BFO}$ is symmetry breaking. However, contrary to the 
earlier phase diagram ${ }^{6}$ the pressure-induced paramagnetic metallic phase is not cubic, but rather of orthorhombic symmetry.

The above discussion and the phase diagram in Fig. 8 are based only on temperature measurement at 1 bar and pressure measurements at $300 \mathrm{~K}$. As a consequence, the unexplored large intermediate $P-T$ region remains hypothetic and is likely to give rise to new and unexpected features. We strongly encourage the exploration of the strain-temperature phase diagram, be it by hydrostatic pressure or strain in thin films, and particularly into the midpressure region with competing phases.

\section{ACKNOWLEDGMENTS}

We are grateful to the ESRF staff, especially M. Mezouar for allocation of inhouse beamtime, D. Chernyshov and A. Bossak for their help with the softwares, and W. Crichton for fruitful discussions. Financial support from the French National Research Agency (ANR Blanc PROPER) is acknowledged. *mael.guennou@supaero.org

†pierre.bouvier@grenoble-inp.fr

${ }^{1}$ R. Ramesh and N. A. Spaldin, Nat. Mater. 6, 21 (2007).

${ }^{2}$ S.-W. Cheong and M. Mostovoy, Nat. Mater. 6, 13 (2007).

${ }^{3}$ M. Fiebig, J. Phys. D: Appl. Phys. 38, R123 (2005).

${ }^{4}$ H. Béa, M. Gajek, M. Bibes, and A. Barthélémy, J. Phys. Condens. Matter 20, 434221 (2008).

${ }^{5}$ J. Kreisel, B. Noheda, and B. Dkhil, Phase Transitions 82, 633 (2009).

${ }^{6}$ G. Catalan and J. F. Scott, Adv. Mater. 21, 2463 (2009).

${ }^{7}$ A. M. Glazer, Acta Crystallogr. Sect. B 28, 3384 (1972).

${ }^{8}$ R. H. Mitchell, Perovskites: Modern and Ancient (Almaz Press, Ontario, Canada, 2002).

${ }^{9}$ A. M. Glazer, Phase Transitions 84, 405 (2011)

${ }^{10}$ P. Ghosez, E. Cockayne, U. V. Waghmare, and K. M. Rabe, Phys. Rev. B 60, 836 (1999).

${ }^{11} \mathrm{~J}$. Junquera and P. Ghosez, Handbook of Theoretical and Computational Nanotechnology (American Scientific, Stevenson Ranch, CA, 2006)

${ }^{12}$ P.-E. Janolin, P. Bouvier, J. Kreisel, P. A. Thomas, I. A. Kornev, L. Bellaiche, W. Crichton, M. Hanfland, and B. Dkhil, Phys. Rev. Lett. 101, 237601 (2008).

${ }^{13}$ J. F. Scott, Phys. Rev. 183, 823 (1969).

${ }^{14}$ P. A. Fleury, J. F. Scott, and J. M. Worlock, Phys. Rev. Lett. 21, 16 (1968).

${ }^{15}$ G. A. Samara, T. Sakudo, and K. Yoshimitsu, Phys. Rev. Lett. 35, 1767 (1975).

${ }^{16}$ P. Bouvier and J. Kreisel, J. Phys. Condens. Matter 14, 3981 (2002).

${ }^{17}$ R. J. Angel, J. Zhao, and N. L. Ross, Phys. Rev. Lett. 95, 025503 (2005).

${ }^{18}$ J. Zhao, N. L. Ross, and R. J. Angel, Acta Crystallogr. Sect. B 62, 431 (2006).

${ }^{19}$ J. Zhao, N. L. Ross, and R. J. Angel, Acta Crystallogr. Sect. B 60, 263 (2004).

${ }^{20}$ T. Tohei, A. Kuwabara, T. Yamamoto, F. Oba, and I. Tanaka, Phys. Rev. Lett. 94, 035502 (2005).

${ }^{21}$ U. D. Venkateswaran, V. M. Naik, and R. Naik, Phys. Rev. B 58, 14256 (1998)

${ }^{22}$ A. K. Sood, N. Chandrabhas, D. V. S. Muthu, and A. Jayaraman, Phys. Rev. B 51, 8892 (1995).

${ }^{23}$ D. Gourdain, P. Pruzan, J. M. Besson, S. Klotz, J. C. Chervin, B. Canny, W. G. Marshall, J. S. Loveday, and M. Hanfland, Phys. Rev. B 65, 054104 (2002).

${ }^{24}$ P. Pruzan, D. Gourdain, and J. C. Chervin, Phase Transitions 80, 1103 (2007).
${ }^{25}$ F. Cerdeira, W. B. Holzapfel, and D. Bäuerle, Phys. Rev. B 11, 1188 (1975).

${ }^{26}$ J. A. Sanjurjo, E. López-Cruz, and G. Burns, Phys. Rev. B 28, 7260 (1983).

${ }^{27}$ J. Kreisel, B. Dkhil, P. Bouvier, and J.-M. Kiat, Phys. Rev. B 65, 172101 (2002).

${ }^{28}$ J. Kreisel, P. Bouvier, B. Dkhil, P. A. Thomas, A. M. Glazer, T. R. Wellberry, B. Chaabane, and M. Mezouar, Phys. Rev. B 68, 014113 (2003).

${ }^{29}$ B. Chaabane, J. Kreisel, B. Dkhil, P. Bouvier, and M. Mezouar, Phys. Rev. Lett. 90, 257601 (2003).

${ }^{30}$ P.-E. Janolin, B. Dkhil, P. Bouvier, J. Kreisel, and P. A. Thomas, Phys. Rev. B 73, 094128 (2006).

${ }^{31}$ A. Sani, B. Noheda, I. A. Kornev, L. Bellaiche, P. Bouvier, and J. Kreisel, Phys. Rev. B 69, 020105 (2004).

${ }^{32}$ J. Rouquette, J. Haines, V. Bornand, M. Pintard, P. Papet, W. G. Marshall, and S. Hull, Phys. Rev. B 71, 024112 (2005).

${ }^{33}$ J. Rouquette, J. Haines, V. Bornand, M. Pintard, P. Papet, C. Bousquet, L. Konczewicz, F. A. Gorelli, and S. Hull, Phys. Rev. B 70, 014108 (2004).

${ }^{34}$ I. A. Kornev and L. Bellaiche, Phase Transitions 80, 385 (2007).

${ }^{35}$ I. A. Kornev, L. Bellaiche, P. Bouvier, P. E. Janolin, B. Dkhil, and J. Kreisel, Phys. Rev. Lett. 95, 196804 (2005).

${ }^{36}$ E. Bousquet and P. Ghosez, Phys. Rev. B 74, 180101 (2006).

${ }^{37}$ R. Haumont, J. Kreisel, and P. Bouvier, Phase Transitions 79, 1043 (2006).

${ }^{38}$ R. Haumont, P. Bouvier, A. Pashkin, K. Rabia, S. Frank, B. Dkhil, W. A. Crichton, C. A. Kuntscher, and J. Kreisel, Phys. Rev. B 79, 184110 (2009).

${ }^{39}$ M. Guennou, P. Bouvier, R. Haumont, G. Garbarino, and J. Kreisel, Phase Transitions 84, 474 (2011).

${ }^{40}$ A. Gavriliuk, V. Struzhkin, I. Lyubutin, M. Hu, and H. Mao, JETP Lett. 82, 224 (2005)

${ }^{41}$ A. G. Gavriliuk, V. Struzhkin, I. S. Lyubutin, I. A. Trojan, M. Y. $\mathrm{Hu}$, and P. Chow, in Materials Research at High Pressure, MRS Proceedings, edited by R. Bini, A. F. Goncharov, R. J. Hemley, and M. R. Manaa (Materials Research Society, Warrendale, PA, 2006), Vol. 987, pp. 147-152.

${ }^{42}$ A. G. Gavriliuk, V. V. Struzhkin, I. S. Lyubutin, and I. A. Troyan, JETP Lett. 86, 197 (2007).

${ }^{43}$ A. A. Belik, H. Yusa, N. Hirao, Y. Ohishi, and E. TakayamaMuromachi, Chem. Mater. 21, 3400 (2009).

${ }^{44}$ Y. Yang, J. Y. Sun, K. Zhu, Y. L. Liu, J. Chen, and X. R. Xing, J. Phys. Condens. Matter 21, 385901 (2009). 
${ }^{45}$ J. L. Zhu, S. M. Feng, L. J. Wang, C. Q. Jin, X. H. Wang, L. T. Li, Y. C. Li, X. D. Li, and J. Liu, High Press. Res. 30, 265 (2010).

${ }^{46}$ M. Guennou, P. Bouvier, J. Kreisel, and D. Machon, Phys. Rev. B 81, 054115 (2010).

${ }^{47}$ M. Guennou, P. Bouvier, B. Krikler, J. Kreisel, R. Haumont, and G. Garbarino, Phys. Rev. B 82, 134101 (2010).

${ }^{48}$ A. G. Gavriliuk, V. V. Struzhkin, I. S. Lyubutin, S. G. Ovchinnikov, M. Y. Hu, and P. Chow, Phys. Rev. B 77, 155112 (2008).

${ }^{49}$ D. P. Kozlenko, A. A. Belik, A. V. Belushkin, E. V. Lukin, W. G. Marshall, B. N. Savenko, and E. Takayama-Muromachi, Phys. Rev. B 84, 094108 (2011).

${ }^{50}$ P. Ravindran, R. Vidya, A. Kjekshus, H. Fjellvåg, and O. Eriksson, Phys. Rev. B 74, 224412 (2006).

${ }^{51}$ H. Feng, J. Magn. Magn. Mater. 322, 3755 (2010).

${ }^{52}$ O. Diéguez, O. E. González-Vázquez, J. C. Wojdeł, and J. Íñiguez, Phys. Rev. B 83, 094105 (2011).

${ }^{53}$ O. E. González-Vázquez and J. Íñiguez, Phys. Rev. B 79, 064102 (2009).

${ }^{54}$ R. Haumont, R. Saint-Martin, and C. Byl, Phase Transitions 81, 881 (2008).

${ }^{55}$ H. K. Mao, J. Xu, and P. M. Bell, J. Geophys. Res. 91, 4673 (1986).

${ }^{56}$ A. P. Hammersley, S. O. Svensson, M. Hanfland, A. N. Fitch, and D. Hausermann, High Press. Res. 14, 235 (1996).

${ }^{57}$ T. Holland and S. Redfern, Mineral. Mag. 61, 65 (1997).

${ }^{58}$ A. Dewaele, P. Loubeyre, and M. Mezouar, Phys. Rev. B 70, 094112 (2004).

${ }^{59}$ A. K. Singh, J. Phys. Chem. Solids 65, 1589 (2004).

${ }^{60} \mathrm{~V}$. Janovec and J. Přívatská, International Tables for Crystallography (Springer, Dordrecht, 2006), Vol. D, Chap. 3.4, pp. 449-505.

${ }^{61}$ J. Zhao, R. J. Angel, and N. L. Ross, J. Appl. Crystallogr. 43, 743 (2010).

${ }^{62}$ E. K. H. Salje, Phase Transitions in Ferroelastic and Co-elastic Crystals (Cambridge University Press, Cambridge, UK, 1993).

${ }^{63}$ D. P. Kozlenko, A. A. Belik, S. E. Kichanov, I. Mirebeau, D. V. Sheptyakov, T. Strassle, O. L. Makarova, A. V. Belushkin, B. N. Savenko, and E. Takayama-Muromachi, Phys. Rev. B 82, 014401 (2010).
${ }^{64}$ M. E. Lines and A. M. Glass, Principles and Applications of Ferroelectrics (Clarendon Press, Oxford, 1977).

${ }^{65}$ B. Noheda, Curr. Opin. Solid State Mater. Sci. 6, 27 (2002).

${ }^{66}$ B. Noheda and D. E. Cox, Phase Transitions 79, 5 (2006).

${ }^{67}$ J. Íñiguez and D. Vanderbilt, Phys. Rev. Lett. 89, 115503 (2002).

${ }^{68} \mathrm{M}$. Guennou et al. (unpublished).

${ }^{69}$ S. K. Mishra, R. Mittal, V. Y. Pomjakushin, and S. L. Chaplot, Phys. Rev. B 83, 134105 (2011).

${ }^{70}$ D. Fu, M. Endo, H. Taniguchi, T. Taniyama, and M. Itoh, Appl. Phys. Lett. 90, 252907 (2007).

${ }^{71}$ A. M. Glazer, Acta Crystallogr. Sect. A 31, 756 (1975).

${ }^{72}$ D. J. Adams and B. Amadon, Phys. Rev. B 79, 115114 (2009).

${ }^{73}$ D. C. Arnold, K. S. Knight, G. Catalan, S. A. T. Redfern, J. F. Scott, P. Lightfoot, and F. D. Morrison, Adv. Funct. Mater. 20, 2116 (2010).

${ }^{74}$ G. K. Rozenberg, M. P. Pasternak, W. M. Xu, L. S. Dubrovinsky, S. Carlson, and R. D. Taylor, Europhys. Lett. 71, 228 (2005).

${ }^{75}$ I. S. Lyubutin, S. G. Ovchinnikov, A. G. Gavriliuk, and V. V. Struzhkin, Phys. Rev. B 79, 085125 (2009).

${ }^{76}$ M. P. Pasternak, W. M. Xu, G. K. Rozenberg, and R. D. Taylor, in Perovskite Materials (Materials Research Society, Warrendale, PA, 2002), Vol. 718, p. 15.

${ }^{77}$ R. Palai, R. S. Katiyar, H. Schmid, P. Tissot, S. J. Clark, J. Robertson, S. A. T. Redfern, G. Catalan, and J. F. Scott, Phys. Rev. B 77, 014110 (2008).

${ }^{78}$ R. Haumont, I. A. Kornev, S. Lisenkov, L. Bellaiche, J. Kreisel, and B. Dkhil, Phys. Rev. B 78, 134108 (2008).

${ }^{79}$ I. A. Kornev, S. Lisenkov, R. Haumont, B. Dkhil, and L. Bellaiche, Phys. Rev. Lett. 99, 227602 (2007).

${ }^{80}$ J. Kreisel, P. Jadhav, O. Chaix-Pluchery, M. Varela, N. Dix, F. Sánchez, and J. Fontcuberta, J. Phys. Condens. Matter 23, 342202 (2011).

${ }^{81}$ W. Siemons, M. D. Biegalski, J. H. Nam, and H. M. Christen, Appl. Phys. Express 4, 095801 (2011).

${ }^{82}$ G. J. MacDougall et al., e-print arXiv:1107.2975 (unpublished).

${ }^{83}$ I. C. Infante et al., e-print arXiv:1105.6016 (unpublished). 